\title{
Identidade de modelos hipsométricos para um plantio de eucalipto clonal
}

\author{
Thaís Schmitt ${ }^{1}$ Valdir Carlos Lima de Andrade ${ }^{1}$ \\ ${ }^{1}$ Universidade Federal do Tocantins, campi Gurupi, Rua Badejos, Lote 7 - Chácaras 69/72, Gurupi-TO, 77402-970 \\ *Author for correspondence: vclandrade@ hotmail.com \\ Received: May 2018 / Accepted: May 2019/ Published: June 2019
}

\section{Resumo}

Este trabalho teve como objetivo definir a melhor forma de ajuste de modelos hipsométricos para eucalipto clonal na região Sul do Estado do Tocantins, e em seguida analisar a acurácia do modelo escolhido, aplicando-o em uma situacão florestal diferente. Os dados foram divididos em dois lotes: um de ajuste, com cinco árvores de cada parcela, onde se estipulou três classes de diâmetro e três classes de altura dominante; e o segundo, de aplicação, com três árvores de cada parcela. Inicialmente, determinou-se o coeficiente de determinação ajustado, erro-padrão da estimativa e análise gráfica residual. Em seguida, empregou-se um teste de identidade de modelos para se verificar o ajuste por classe ou com todos os dados. No final da análise, para avaliar os modelos em um teste de validação, foram utilizados os critérios: soma de quadrados do resíduo relativo, raiz quadrada do erro médio, erro médio percentual. Concluiu-se que as melhores formas de ajuste tanto para o modelo local quanto para o regional é o de realizar o ajuste por classe de altura dominante, sendo o modelo regional o de melhor performance.

Palavras-chave: Teste de identidade, Regressão linear, Validação.

Abstract

This work had as objective to define the best form of adjustment of hypsometric models for clonal eucalyptus in the South of Tocantins, and after that analyze the accuracy of the chosen model, applying it in adifferent forest situation. The data were divided in two lots: one, setting, with five trees of each plot, which stipulated three classes of diameter and three classes of dominant height; and the second, of application, with three trees of each plot. Initially, the coefficient of determination adjusted, standard error of the estimate, and residual graphical analysis were determined. Then, a model identify test is carried out to verify or adjust by class or with all the data. At the end of the analysis, to evaluate the stability of the models in a validation test, the following criteria were used: sum of squares of he residual residue, square root mean error mean error. It was concluded that the best forms of adjustment for both the local and the regional model were to perform an adjustment by class, with the regional model being the most appropriate to use.

Keywords: Identity test, Linear regression, Validation.

\section{Introdução}

O Eucalyptus é um dos principais gêneros utilizados nos plantios florestais brasileiros devido à sua alta produtividade e diversidade de utilização. Há algumas décadas, muitos estudos foram direcionados a este gênero sob diferentes aspectos, dentre os quais, destaca-se a relação hipsométrica conforme alguns estudos, por exemplo, conduzidos por: Batista et al. (2001), Leite e Andrade (2003), Tomé et al. (2007), Ribeiro et al. (2010),
Oliveira et al. (2011), Trautenmüller et al. (2014), Sousa et al. (2017).

Uma relação hipsométrica trata da caracterização do relacionamento da altura das árvores com algumas variáveis explicativas representativas dos efeitos do local, ou, nível de produtividade (altura dominante, sítio), do tempo (idade), da competição (área basal, número de árvores), dentre outras. Isso se faz necessário, porque é feita a medição da altura de parte das árvores das parcelas de um inventário florestal, ficando algumas árvores sem a medição desta variável. Por isso, gera-se uma equação hipsométrica adequada à predição da altura das árvores em que se mediu apenas o seu diâmetro (Batista et al. 2001; Leite e Andrade 2003; Bartoszeck et al. 2004; Machado et al. 2008; Ribeiro et al. 2010; Oliveira et al. 2011; Andrade et al. 2015; Sena et al. 2015; Santos et al. 2016; Soares et al. 2017).

Devido as diferentes situações que encontra-se o plantio florestal (idade, manejo, solos, etc), é preciso definir a melhor forma para o ajuste do modelo hipsométrico, por exemplo, por classe de diâmetro, por classe de altura dominante, por idade, ou, um só ajuste é necessário, independente da situação caracterizada (Bartoszeck et al. 2004; Ribeiro et al. 2010; Vendruscolo et al. 2015; Andrade et al. 2015). Esta situação, define-se em saber se o conjunto de equações ajustadas é idêntica, ou seja, se o caso em estudo pode ser representado por uma única equação, cabendo-se testar a identidade de modelos (Regazzi e Silva 2010).

Diante deste contexto, este estudo teve como objetivo avaliar o ajuste e testar a identidade de modelos hipsométricos empregando-se diferentes formas de agrupamento de dados da relação hipsométrica de eucalip to clonal.

\section{Material e Métodos}

Coleta de dados e modelos hipsométricos ajustados

Este estudo foi realizado com dados coletados em 75,6 ha de um plantio clonal do híbrido entre Eucalyptus camaldulensis Dehnh e Eucalyptus spp. (E1277) localizado na região Sul do Tocantins, próximo ao município de Gurupi (Figura 1). Nesta região. a altitude média é de $225 \mathrm{~m}$ com clima predominante tropical de estação seca (Classificação climática de Köppen-Geiger: Aw). 


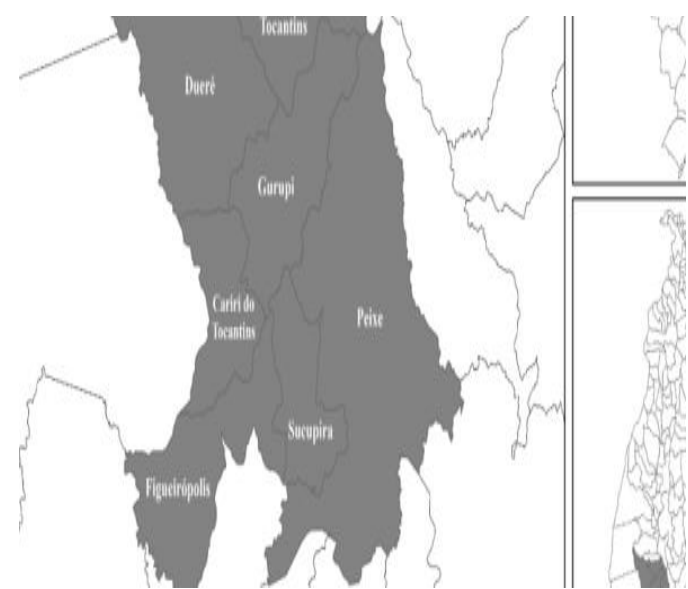

Figura 1. Localização da área de estudo na Região Sul do Estado do Tocantins. (Fonte: map sofworld, adaptado).

Os dados foram coletados em 11 parcelas retangulares de $348 \mathrm{~m}^{2}$ cada, as quais constituem um inventário florestal contínuo. Em cada parcela. mediu-se o diâmetro de todas as árvores, a altura total das oito primeiras árvores para amostrar a relação hipsométrica e de quatro árvores dominantes para obter a altura média das 100 árvores de maior diâmetro por hectare. O plantio foi feito no espacamento inicial de $6.0 \times 1.5 \mathrm{~m}$ e tinha cerca de 3 anos de idade na ocasião da medição das parcelas.

As informações obtidas no inventário florestal, quanto à relação hisométrica, foram divididas em dois bancos de dados. O primeiro. contendo as cinco primeiras árvores de cada parcela, totalizou 55 árvores utilizadas no ajuste de dois modelos hipsométricos com os dados agrupados em três classes de diâmetro $(C D)$ e de altura dominante (CHd). Já, o segundo banco de dados, que se constituiu das três árvores restantes de cada parcela. totalizou 33 árvores para serem utilizadas em um teste de validação com dados independentes (Batista et al. 2001; Tomé et al. 2007; Santos et al. 2016).

Dentre os inúmeros modelos hipsométricos existentes, foram selecionados os dois mais testados vara plantios com Eucalyptus no Brasil, sendo um de característica local, onde há apenas o diâmetro como variável independente e, outro de característica regional, que envolve também a altura dominante como variável independente (Leite e Andrade 2003; Tomé et al. 2007; Machado et al. 2008; Andrade et al. 2015). Portanto, os modelos hipsométricos utilizados, foram os modelos de Curtis (1967) e o de Campos et al. (1986), ambos modelo local (1) e modelo regional (2), respectivamente, quais sejam os seguintes:

$$
\begin{aligned}
& \operatorname{Ln}(H)=b_{0}+b_{1}\left(\frac{1}{D}\right)+\operatorname{Ln}(\varepsilon) \\
& \operatorname{Ln}(H)=b_{0}+b_{1}\left(\frac{1}{D}\right)+b_{2} \operatorname{Ln}(H d)+\operatorname{Ln}(\varepsilon)
\end{aligned}
$$

Em que: $\operatorname{Ln}(\mathrm{H})=$ logaritmo neperiano da altura; $\mathrm{b}_{0}, \mathrm{~b}_{1} \mathrm{e}$ $\mathrm{b}_{2}=$ coeficientes dos modelos a serem estimados; $\mathrm{D}=$ diâmetro a altura do peito $(1,3 \mathrm{~m}) ; \mathrm{Ln}(\mathrm{Hd})=$ logaritmo neperiano da altura dominante; $\varepsilon=$ erro do modelo.

\section{Critérios estatísticos}

Inicialmente, os modelos 1 e 2 foram submetidos a uma análise por meio dos critérios estatísticos: coeficiente de determinação ajustado, erro padrão da estimativa e análise gráfica dos resíduos. O emprego desses critérios estatísticos tiveram o intuito de verificar, preliminarmente, sobre a superioridade de um modelo frente ao outro quanto ao ajuste aos dados da relação hipsométrrica. Ressalta-se que, tais critérios estatísticos, foram utilizados conforme feito por Batista et al. (2001), Machado et al. (2008), Oliveira et al. (2011), Trautenmüller et al. (2014), Vendruscolo et al. (2015) e Santos et al. (2016).

$\mathrm{O}$ teste de identidade de modelos também foi utilizado (Tabela 1). Esse teste, descrito por Graybill (1976) foi utilizado para constatar diferença significativa entre as regressões ajustadas para cada classe de diâmetro e classe de altura dominante (modelo completo) e a regressão ajustada englobando todas as classes juntas (modelo reduzido), conforme relatam: Leite e Andrade (2003), Regazzi e Silva (2010), Ribeiro et al. (2010), Andrade et al. (2015).

Tabela 1. Teste de identidade dos modelos para verificar a hipótese de que o modelo reduzido não apresenta diferença

\begin{tabular}{|c|c|c|c|}
\hline $\begin{array}{l}\text { Fonte } \\
\text { Variação }\end{array}$ & GL & SQ & QM \\
\hline $\begin{array}{l}\text { Modelo Completo } \\
\text { (Comp) }\end{array}$ & $(\mathrm{CP})$ & SQComp & \\
\hline $\begin{array}{l}\text { Modelo Reduzido } \\
\text { (Red) }\end{array}$ & $\mathrm{P}$ & SQRed & \\
\hline $\begin{array}{l}\text { Diferença para } \\
\text { Testar hipóteses } \\
\text { (Dif) }\end{array}$ & $\begin{array}{l}(\mathrm{CP})- \\
\mathrm{P}\end{array}$ & $\begin{array}{l}\text { SQComp- } \\
\text { SQRed }\end{array}$ & $\begin{array}{l}\text { (SQC- } \\
\text { SQR)/(CP- } \\
\text { P) }\end{array}$ \\
\hline Resíduos (Res) & $\begin{array}{l}\mathrm{N}- \\
(\mathrm{CP})\end{array}$ & & $\begin{array}{l}\text { QT - } \\
\text { QC)/(N- } \\
\mathrm{P})\end{array}$ \\
\hline Total (Tot) & $\mathrm{N}$ & SQT ot & - \\
\hline \multicolumn{4}{|c|}{$\begin{array}{l}\text { Em que: } \mathrm{FV}=\text { fonte de variação, } \mathrm{GL}=\text { grau de liberdade; } \mathrm{SQ}=\mathrm{sor} \\
\text { quadrados; } \mathrm{QM}=\text { quadrado médio; } \mathrm{FG}=\text { teste } \mathrm{F} \text { de Graybill; } \mathrm{C}= \\
\text { classes; } \mathrm{P}=\mathrm{n}^{\circ} \text { de coeficientes estimados; } \mathrm{N}=\mathrm{n}^{\circ} \text { total de } \\
\text { SQComp=soma de quadrados do modelo completo, SQRed=so } \\
\text { quadrados do modelo reduzido, SQTot=soma de quadrados } \\
\text { QMDif=quadrado médio da diferença, QMRes=quadrado médi } \\
\text { resíduns } \\
\text { Os modelos na sua forma completa são represen } \\
\text { pelos modelos } 3 \text { e } 4 \text {, cuia forma reduzida é represe } \\
\text { pelos modelos } 1 \text { e } 2 \text {, respectivamente. Portant } \\
\text { modelos completos ajustados foram os seguintes: }\end{array}$} \\
\hline \multicolumn{4}{|c|}{$\begin{array}{l}\text { Modelo completo } 1 \text { : } \\
\operatorname{Ln}(H)=b_{1}(D 1)+b_{2}\left(\frac{D}{D}\right)+b_{3}(D 2)+b_{4}\left(\frac{D 2}{D}\right)+ \\
b_{5}(D 3)+b_{6}\left(\frac{D 3}{D}\right)+\operatorname{Ln}(\varepsilon)\end{array}$} \\
\hline
\end{tabular}
estatística em relação ao modelo completo.

Modelo completo 2:

$$
\begin{aligned}
& \operatorname{Ln}(H)=(\beta 1 D 1)+\beta 2\left(\frac{1}{D}\right) D 1+\beta 3(\text { LnHd }) D 1+ \\
& (\beta 4 D 2)+\beta 5\left(\frac{1}{D}\right) D 2+\beta 6(\text { LnHd }) D 2+(\beta 7 D 3)+ \\
& \beta 8\left(\frac{1}{D}\right) D 3+\beta 9(\text { LnHd }) D 3+\varepsilon
\end{aligned}
$$

Em que: [D1 D2 D3 $]=\left[\begin{array}{lll}1 & 0 & 0\end{array}\right]$ para a classe 1 de CD ou CHd; [D1 D2 D3 $]=\left[\begin{array}{lll}0 & 1 & 0\end{array}\right]$ para a classe 2 de CD ou CHd; [D1 D2 D3 $]=\left[\begin{array}{lll}0 & 0 & 1\end{array}\right]$ para a classe 3 de CD ou $\mathrm{CHd}$.

$\mathrm{Na}$ complementação das análises, realizou-se um teste de validação, adotando-se os critérios estatísticos: soma de quadrados dos resíduos relativos (SQRR), raiz quadrada do erro médio (RQEM) e erro médio percentual (EMP), conforme descritos por: Batista et al. (2001), Tomé et al. (2007), Santos et al. (2016). 


\section{Resultados e Discussão}

Dados de ajuste

Os resultados do ajuste dos modelos reduzidos 1 e 2 (Tabela 2), empregando-se os dados das 55 árvores, proporcionaram uma diferença expressiva entre o coeficiente de detrminação ajustado $\left(R^{2} a j\right)$ e o erro padrão da estimativa (EPE). No modelo 1, obteve-se valores de 0,56 e de $\pm 5,04 \%$ para $R^{2}$ aj e EPE, respectivamente. Já, no modelo 2 , estes valores obtidos foram de 0,67 e de $\pm 4,37 \%$, respectivamente. Observa-se, então, que considerando os critérios $R^{2}$ aj e EPE, o modelo regional (Modelo 2) se sobrepõe ao modelo local (Modelo 1), resultando em melhor ajuste aos dados de relação hip sométrica.

Tabela 2. Resultados obtidos no ajuste dos modelos reduzidos local e regional utilizando-se os dados de ajuste.

\begin{tabular}{llllll}
\hline Modelo & $\boldsymbol{\beta}_{\mathbf{0}}$ & $\boldsymbol{\beta}_{\mathbf{1}}$ & $\boldsymbol{\beta}_{\mathbf{2}}$ & $\mathbf{R}^{\mathbf{2} a j}$ & $\mathbf{E P E}$ \\
\hline Local (1) & 3,29717 & $-6,03541$ & - & 0,56 & 5,04 \\
Regional (2) & 2,02733 & $-4,48918$ & 0,40595 & 0,67 & 4,37 \\
\hline Em que: $\beta 0, \beta 1$ & e $\beta 2=$ coeficientes estimados por regressão linear, Rªj= \\
coeficiente de determinação ajustado e EPE= erro padrão da estimativa (\%).
\end{tabular}

Para o critério $\mathrm{R}^{2} \mathrm{aj}$, os valores encontrados foram semelhantes aos de Souza et al. (2017), sendo considerados satisfatórios para a modelagem da relação hipsométrica. O mesmo ocorreu para EPE, onde Souza et al. (2017) encontraram resultados semelhantes para o modelo 1 em Eucalyptus. Em Ribeiro et al. (2010) e Oliveira et al. (2011), nota-se a demonstração da eficiência deste modelo no estudo da relação hip sométrica para Eucalyptus.

A análise gráfica dos resíduos (Figura 2) permitiu verificar que o modelo com menor variação e distribuição residual mais homogênea foi o modelo regional, confirmando a sua superioridade conforme detectado pelos resultados dos critérios $\mathrm{R}^{2}$ aj e EPE.

$\mathrm{O}$ teste de identidade de modelos (Tabela 3) indicou não haver diferença significativa quando se tem os dados agrupados por classe de diâmetro (CD), tanto para o modelo local $\left(\mathrm{FG}=0,43^{\mathrm{ns}}\right)$, quanto para o regional $\left(\mathrm{FG}=0,53^{\text {ns }}\right)$. Com isso, deve-se optar pelo ajuste do modelo reduzido, isto é, não é necessário dividir os dados em $\mathrm{CD}$, ou seja, pode-se obter uma única equação hipsométrica utilizando-se todas as informações em um único lote de dados.

Tabela 3. Resultados do teste de identidade de modelos por classe de diâmetro e por altura dominate

\begin{tabular}{lll}
\hline Modelo/tipo de agrupamento & SQ (diferença) & FG \\
\hline Local / CD & 0,00594 & $0,43^{\mathrm{ns}}$ \\
\hline Local / CHd & 0,03504 & $3,21^{*}$ \\
\hline Regional / CD & 0,00757 & $0,53^{\mathrm{ns}}$ \\
\hline Regional / CHd & 0,03764 & $3,87^{*}$ \\
\hline Em que: SQ= Soma de quadrados; F= estatística F; $*$ *significativo; ns \\
=não-significativo; Local CD / CHd= modelo local por classe de \\
diâmetro / altura dominante; Regional CD / CHd =modelo regional por \\
classe de diâmetro / altura dominante.
\end{tabular}

Quanto as classes de altura dominante (CHd), houve diferença significativa com o Teste FG para ambos os modelos local $\left(\mathrm{FG}=3,21^{*}\right)$ e regional $\left(\mathrm{FG}=3,87^{*}\right)$, indicando que não é ideal utilizar uma única equação, mas, proceder ao ajuste de uma equação para cada $\mathrm{CHd}$, ou seja, obter três equações hipsométricas em separado para cada classe de altura dominante.

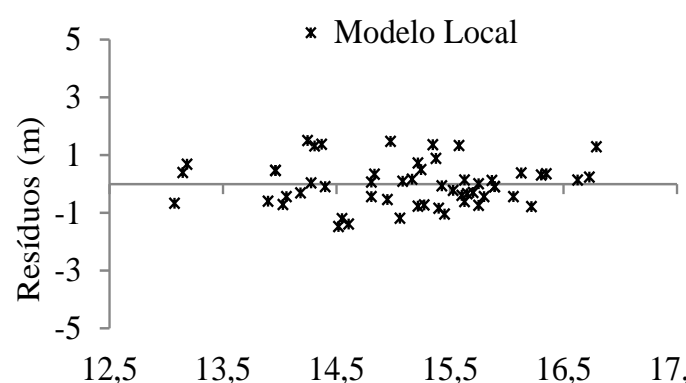

Altura estimada (m)

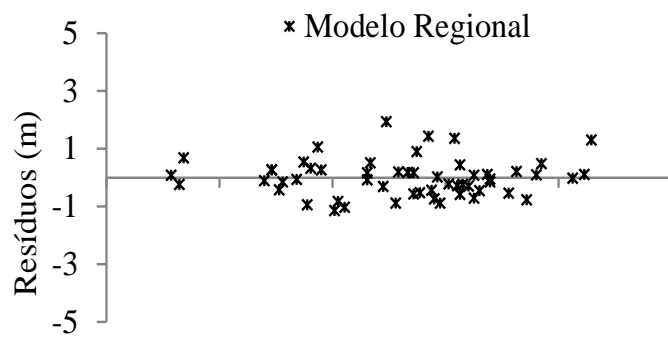

$12,5 \quad 13,5 \quad 14,5 \quad 15,5 \quad 16,5 \quad 17,5$

Altura estimada (m)

Figura 2. Dispersão do erro $(\mathrm{m})$ em relação da alt ura estimada (m) obtida pelo ajuste dos modelos reduzidos local (1) e regional (2) utilizando-se os dados das 55 árvores.

\section{Teste de validação}

Diante das opções de ajuste obtidas, modelo reduzido e modelo completo tendo o ajuste por $\mathrm{CHd}$, realizou-se um teste de validação empregando-se as 33 árvores com dados independentes, cujos resultados estão na Tabela 4. Nota-se, pelo resultado de SQRR, que houve menores valores para os modelos local e regional por classe HD, indicando menores resíduos relativos, com valores de 0,0012 para o local $\mathrm{CHd}$, e 0,0003 para o regional $\mathrm{CHd}$, onde este último foi o de melhor resultado.

Tabela 4. Resultados dos critérios estatísticos utilizados no teste de validação com 33 árvores.

\begin{tabular}{lllll}
\hline & Lred & Rred & LCHd & RCHd \\
\hline SQRR & 0,0035 & 0,0034 & 0,0012 & 0,0003 \\
RQEM & 1,14 & 0,92 & 0,90 & 0,61 \\
EMP & 4,42 & 2,86 & 2,85 & 1,19 \\
\hline
\end{tabular}

Em que: $S Q R R=$ soma de quadrados dos resíduos relativos; RQEM= raíz quadrada do erro médio; EMP = erro médio percentual; Lred=modelo local na forma reduzida; Rred=modelo regional na forma reduzida; LCHd= modelo local com ajuste para cada classe de altura dominante; $\mathrm{RCHd}=$ modelo regional com ajuste para cada classe de altura dominante.

Por outro lado, para RQEM, a opção selecionada para o modelo regional por $\mathrm{CHd}$ se sobressaiu aos demais com valor de $\pm 0,61$. Os modelos locais por $\mathrm{CHd}$ apresentaram valores de RQEM de $\pm 1,14$ para o modelo local reduzido e $\pm 0,90$ para o modelo local por CHd. Já, para EMP, o modelo regional por $\mathrm{CHd}$ apresentou o menor valor $(1,19 \%)$, sendo o maior erro médio obtido pelo modelo local reduzido, com o valor de 4,42\% (Tabela 4).

Diante da análise feita anteriormente, evidencia-se superioridade do modelo regional por $\mathrm{CHd}$ ( $\mathrm{RCHd}$ ), que proporcionou os melhores resultados em todos os critérios considerados. Inclusive, na análise da distribuição de resíduos (Figura 3), onde se nota uma melhor distribuição dos resíduos com tendência mais homogênea, indicando melhor desempenho no seu uso. No modelo reduzido regional, há maior variação para 
árvores com altura até 16 metros, porém, menor quando comparado ao modelo local tanto reduzido como regional.
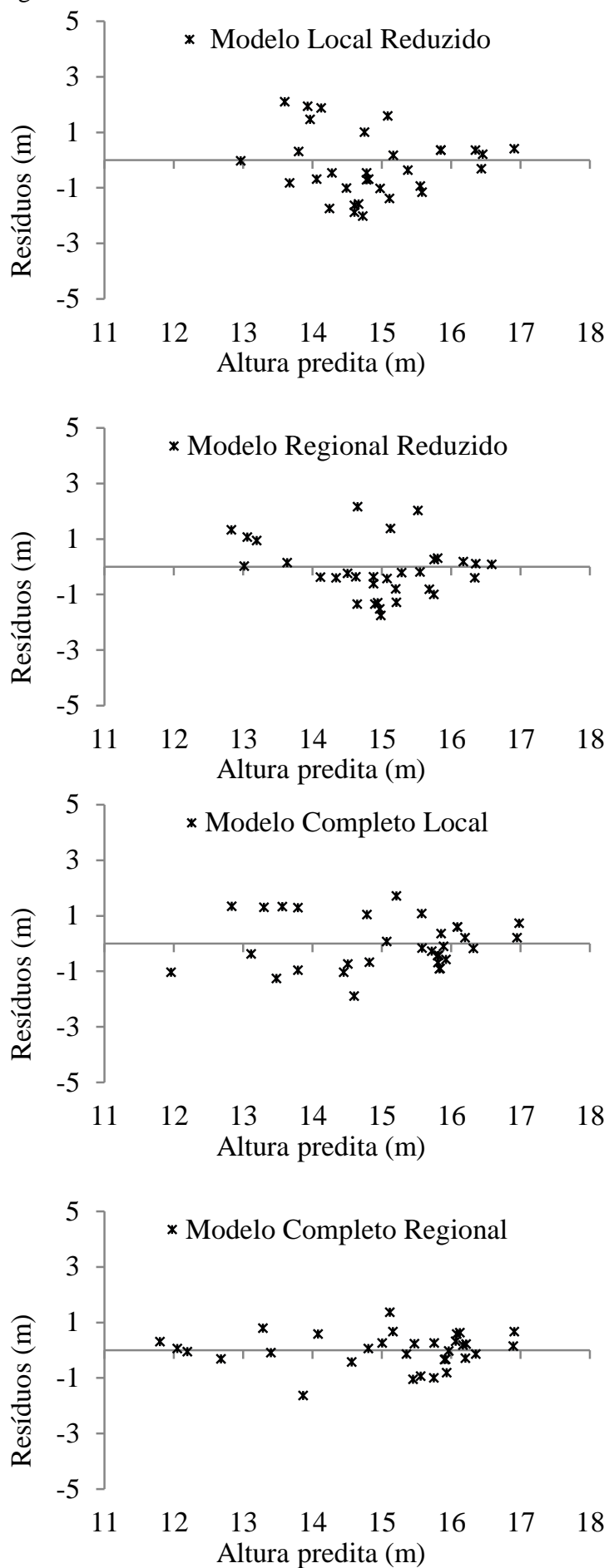

Figura 3. Dispersão do erro $(\mathrm{m})$ em relação da altura estimada(m) obtida em um teste de validação dos modelos reduzidos e completos utilizando-se os dados das 33 árvores independentes.

\section{Conclusões}

Por meio do teste de identidade de modelos concluiuse que se deve optar pelo ajuste por classe de altura dominante, tanto para o modelo local quanto para o modelo regional, ou seja, preferir o ajuste do modelo completo. Já, para o agrupamento por classe de diâmetro, a melhor opção é o ajuste de uma equação, tanto para o modelo local quanto para o regional com o ajuste do modelo reduzido.

No teste de validação, ocorreu superioridade do modelo regional por $\mathrm{CHd}$ em todos os critérios estatísticos analisados, onde o ajuste foi realizado para cada classe separadamente, sendo esta a melhor forma de ajuste para os dados desse plantio clonal de Eucalyptus. Pode-se concluir, também, que a decisão obtida pelo teste de identidade de modelos de Graybill, foi compatível pela decisão tomada com os critérios estatísticos de $\mathrm{R}^{2} \mathrm{aj}$, $\mathrm{s}_{\mathrm{yx}}$, análise gráfica da distribuição dos resíduos e, além disso, houve a mesma decisão no teste de validação.

\section{Agradecimentos}

Agradecimentos são dispensados à CAPES pela concessão da bolsa de pesquisa para desenvolver esta pesquisa.

\section{Referências}

Andrade VCL, Kroetz EA, Nicola A, Souza PB, Nohama FK, Leite HG, Binoti DHB, Binoti MLMS (2015). Amostragem e agrupamento de dados de relação hipsométrica em inventários florestais de Cerrado Tocantinense. Pesquisa Florestal Brasileira, 35(83):227238. doi:10.4336/2015.pfb.35.83.683.

Bartoszeck ACPS, Machado SA, Figueiredo-Filho AF, Oliveira EB (2004) Dinâmica da relação hipsométrica em função da idade, do sítio e da densidade inicial de povoamentos de bracatinga da região metropolitana de Curitiba, Paraná. Revista Árvore, 28(4):517-533. doi:10.1590/S0100-67622004000400006.

Batista JLF, Couto HTZ, Marquesini M (2001) Desempenho de modelos de relações hipsométricas: estudo em três tipos de floresta. Scientia Forestalis, 60:149-163.

Graybill J (1976) Theory and application of the linear model. Belmont: Duxbury.

Leite HG, Andrade VCL (2003) Importância das variáveis altura dominante e altura total em equações hipsométricas e volumétricas. Revista Árvore, 27(3):301310. doi:10.1590/S0100-67622003000300005.

Machado SA, Nascimento RGM, Augustynczik ALD, Silva LCR, Figura MA, Pereira EM, Téo SJ (2008) Comportamento da relação hipsométrica de Araucaria angustifolia no capão da Engenharia Florestal da UFPR. Pesquisa Florestal Brasileira, (56):5-16.

Oliveira FGRB, Sousa GTO, Azevedo GB, Barreto PAB (2011) Desempenho de modelos hipsométricos para um povoamento de Eucalyptus urophylla no município de Jaguaquara, Bahia. Enciclopédia Biosfera - Centro Científico Conhecer, 7(13):331-338.

Regazzi AJ, Silva CH (2010) Testes para verificar a igualdade de parâmetros e a identidade de modelos de regressão não-linear em dados de experimento com delineamento em blocos casualizados. Ceres, 57(3):315320 .

Ribeiro A, Filho ACF, Mello JM, Ferreira MZ, Lisboa PMM, Scolforo JRS (2010) Estratégias e metodologias de ajuste de modelos hipsométricos em plantios de 
Eucalvptus sp. Cerne, 16(1):22-31. doi:10.1590/S010477602010000100003 .

Santos MJF, Andrade VCL, Souza PB (2016) Amostragem relativa de dados da relação hipsométrica de cerrado tocantinense. Revista Brasileira de Biometria, 34(4):633-645.

Sena ALM, Silva-Neto AJ, Oliveira GMV, Calegario N (2015) Modelos lineares e não lineares com uso de covariantes para relação hipsométrica de duas espécies de pinus tropicais. Ciência Florestal, 25(4):969-980. doi:10.5902/1980509820651.

Soares KL, Costa LS, Araújo MS, Calixto-Júnior JED, Interamnense MT (2017) Modelagem hipsométrica de Eucalyptus grandis W. Hill ex Maiden. no sudeste do estado de Goiás. Revista de Agricultura Neotropical, 4(1):51-57.

Souza AS, Santos JX, Souza DV (2017) Modelagem da relação hipsométrica para um povoamento híbrido de eucalipto na Amazônia Brasileira. Biofix scientific journal, 2(1):44-53. doi:10.5380/biofix.v2i1.51395.

Tomé M, Ribeiro F, Faias S (2007) Relação hip sométrica geral para Eucalyptus globulus Labill. em Portugal. Silva Lusitana, 15(1):41-55.

Trautenmüller JW, Balbinot R, Gonzatto GL, Watzlawick LF, Vendruscolo R (2014) Relação hipsométrica em floresta estacional decidual. Enciclopédia Biosfera Centro Científico Conhecer, 10(19):1633-1641.

Vendruscolo DGS. Chaves AGS. Silva RS. Souza HS. Medeiros RA, Motta AS, Silva FT (2015) Identidade em Modelos Hipsométricos para Tectona grandis com Diferentes Espaçamentos em Cáceres-MT. Nativa, 3(3):44-49. doi:10.14583/2318-7670.v03n01a07. 